\title{
Measurement of Trace Water Vapor in a Carbon Dioxide Removal Assembly Product Stream
}

\author{
Joda Wormhoudt, Joanne H. Shorter, J. Barry McManus, David D. Nelson, \\ Mark S. Zahniser, and Andrew Freedman \\ Aerodyne Research, Inc. \\ Melissa Campbell \\ Hamilton Sundstrand \\ Clarence T. Chang \\ NASA Glenn Research Center \\ Frederick D. Smith \\ NASA Johnson Space Center
}

Copyright (C) 2004 SAE International

\begin{abstract}
The International Space Station Carbon Dioxide Removal Assembly (CDRA) uses regenerable adsorption technology to remove carbon dioxide $\left(\mathrm{CO}_{2}\right)$ from cabin air. Product water vapor measurements from a CDRA test bed at the NASA Marshall Space Flight Center were made using a tunable infrared diode laser differential absorption spectrometer (TILDAS) provided by NASA Glenn Research Center. The TILDAS instrument exceeded all the test specifications, including sensitivity, dynamic range, time response, and unattended operation. During the $\mathrm{CO}_{2}$ desorption phase, water vapor concentrations as low as 5 ppmv were observed near the peak of $\mathrm{CO}_{2}$ evolution, rising to levels of $\sim 40$ ppmv at the end of a cycle. Periods of high water concentration (>100 ppmv) were detected and shown to be caused by an experimental artifact. Measured values of total water vapor evolved during a single desorption cycle were as low as $1 \mathrm{mg}$.
\end{abstract}




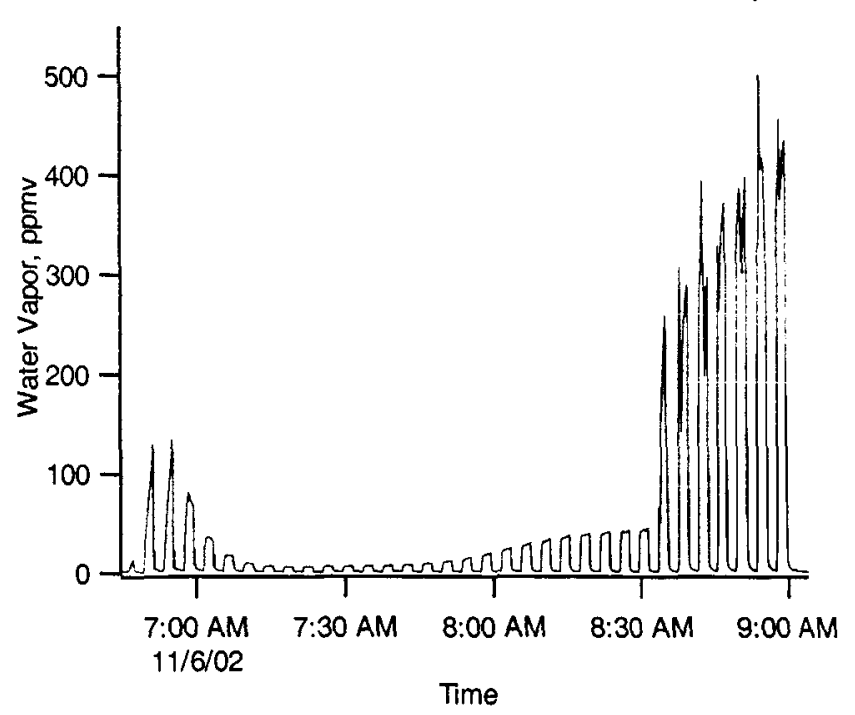

Figure 8. Example water vapor record for one CDRA cycle with normal day/night cycle off.

condensation in the pump. Thus, understanding the source of this high concentration phase and finding a means of suppressing it became the focus of subsequent testing

Figure 9 shows the results when the normal day/night cycle was superimposed on two $\mathrm{CO}_{2}$ desorption cycles. During the first CDRA cycle, the heater on the scrubber bed was turned off midway through the normal desorption cycle. Compared to the data in Figure 8, there is an extra burst of high water vapor at $~ 10: 30$ A.M. which coincides with the bed heater turning off and the $\mathrm{CO}_{2}$ flow diminishing rapidly. An overnight record is shown in Figure 10. It can be determined that the

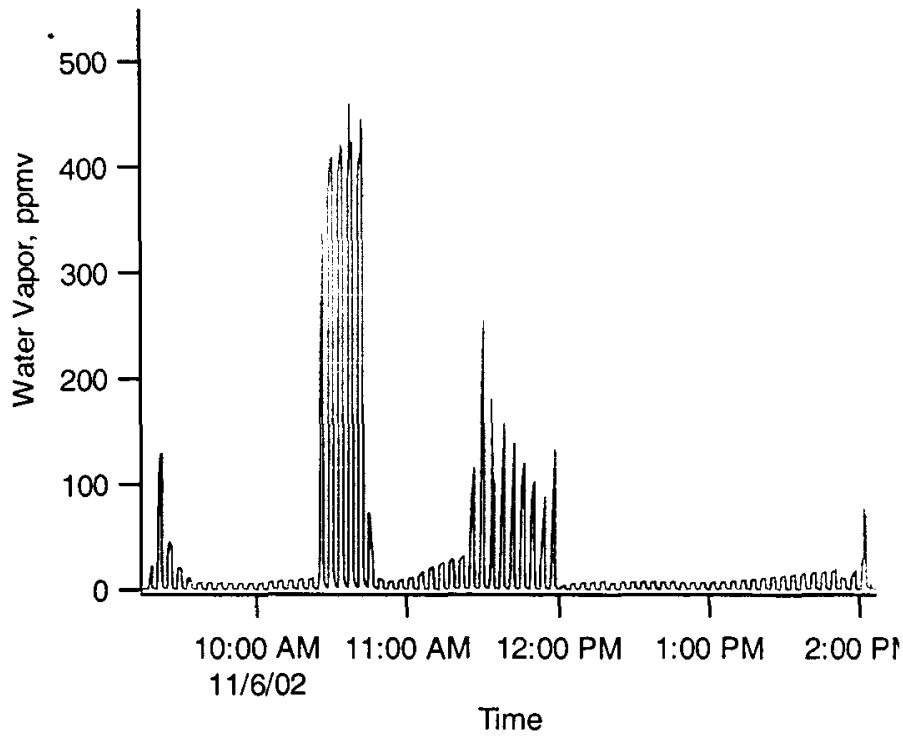

Figure 9. Water vapor record for first cycle with day/night operation.

periods of highest water vapor concentration, roughly constant at about $400 \mathrm{ppmv}$, are observed during the night segments of the 90 minute day/night cycle, when the $\mathrm{CO}_{2}$ desorption rate is low (but only when the space vacuum is valved off). During these low-flow high water concentration events, the CDRA desorption rate and pressure are low, but the TILDAS sample flow rate is still high enough that the measured values are valid.

Possible sources of these high concentration periods included desorption from the bed itself or other regions of the CDRA, leaks of ambient air into the CDRA, and backstreaming through the pumping line. The first possibility appeared to be unlikely as it became clear that

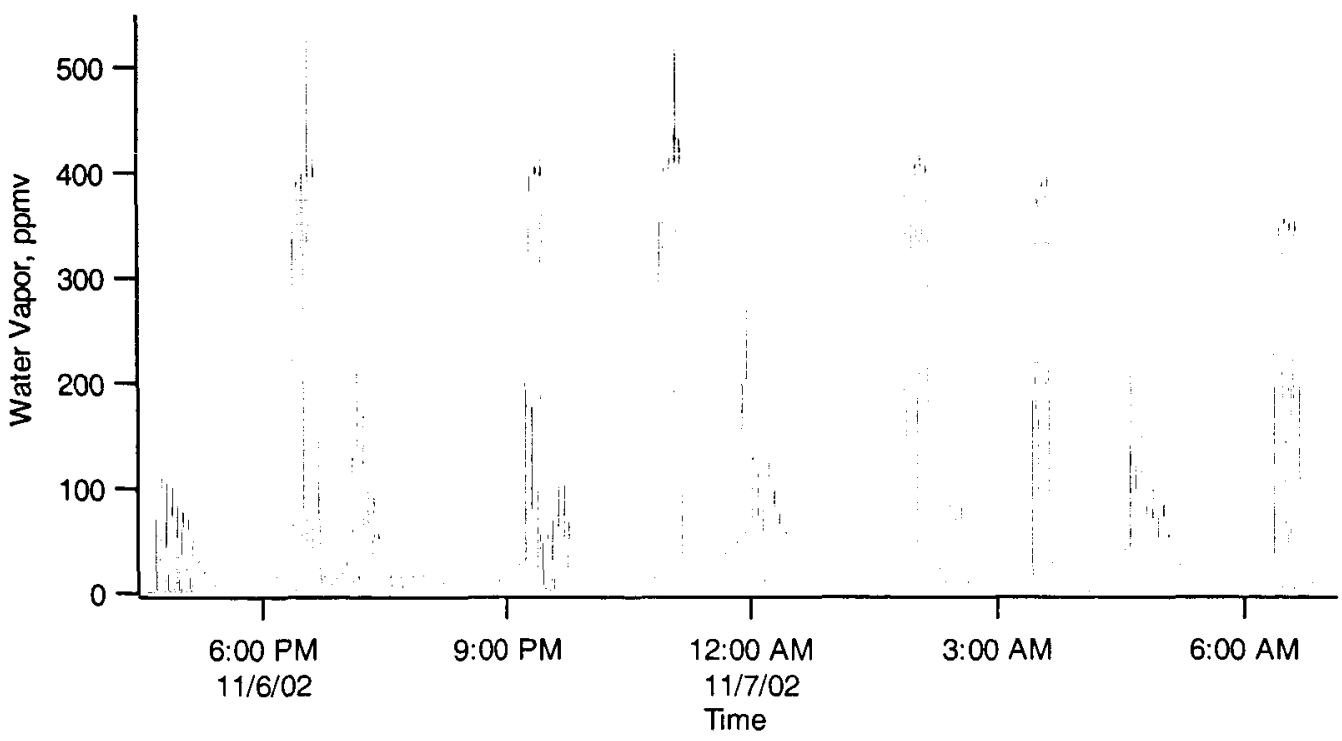

Figure 10. Water vapor record for overnight run with normal solar cycle. 
the high concentration episodes were correlated with low flow periods but not with the progress through the desorption cycle or with bed temperature.

In order to test the hypothesis that a leak into the CDRA was the source of the high water concentration sample, the area around the CDRA was purged with dry facility air. However, the data showed identical concentration profiles to that obtained in Figure 9 . The high concentration spikes were still exactly the same, suggesting that air leaks into the CDRA during low flow periods were not the source.

At this point, the CDRA valving logic was changed so that the isolation valve (V3 in Figure 4) was closed whenever the CDRA was in the night portion of its day/night cycle, thus isolating the measurement system from the mechanical compressor simulator. During the night, the bed heaters are off and the CDRA desorption rate and pressure drop to low values. The result of this change, seen in Figure 11, was that there was no period when high (400 ppmv) water concentrations were seen. Further cycles in this "isolate night" mode are seen in Figure 12. Comparing Figures 10 and 12, the high water concentrations associated with no-flow conditions are reproduced almost exactly, while the low-flow, 400 ppmv episodes are almost completely suppressed.

Once the source of the high humidity spikes were determined to originate from the mechanical compressor simulator, the simulator was carefully examined for a source of high ambient humidity. It was determined that the pump bypass valve (the needle valve in parallel with the diaphragm and piston pumps called out in Figure 4) had been left slightly open. In periods of low or no flow, this open valve would allow backstreaming of ambient air

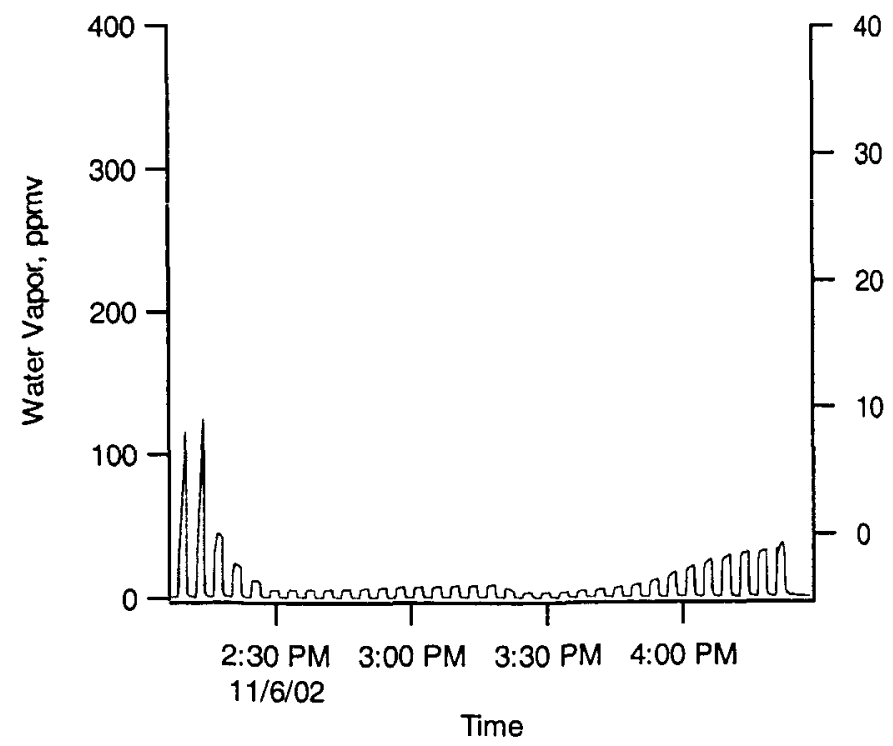

Figure 11. Water vapor record for first cycle with pump isolated at night (the upper curve goes with the right hand, expanded axis). into the system. Once the valve was closed, the data trace in Figure 13 was generated. Although there is still a period of higher water concentration associated with the low-flow period at the end of the desorption phase, it now occurs later and reaches a much lower value than observed previously. At the end of the 2 minute period during which this rise to 200 ppmv was observed, the space vacuum pump came on and the manual three-way valve at the CDRA was switched to the dry air source. Therefore, no-flow high concentrations are not seen in the period around noon. This run provides strong evidence that the major source for high water vapor concentrations during low CDRA flow periods was indeed backstreaming from this pumping line.

Water concentration data like those shown in Figure 13 can be used to calculate the total water vapor evolution of the CDRA system by multiplying by the total gas flow. The high humidity points, which correspond to low flow conditions, contribute little to the total water vapor flux. In the case of Figure 13, the evolution of water from 10:00 to $11: 10$ am is about $1 \mathrm{mg}(1.5 \mathrm{scc})$.

\section{CONCLUSION}

The gaseous output of the Carbon Dioxide Removal Assembly (CDRA) was sampled in order to measure the amount of water vapor generated and to determine if water vapor concentrations reached sufficiently high levels during the desorption cycle to interfere with attempts to recycle carbon dioxide. In order to accomplish this task, the NASA Glenn tunable infrared diode laser differential absorption spectrometer (TILDAS) instrument was configured for the measurement of trace concentrations of water vapor. This instrument was shown to meet or exceed all the specifications for this test, including sensitivity, time response, and the ability to run unattended for 24 hour periods. Its good sensitivity and time resolution, high dynamic range (over four orders of magnitude), firstprinciples calibration, excellent stability, and ability to display and analyze data in real time, were all critical to the success of the test. During the desorption phase, water vapor concentrations were as low as 5 ppmv near the peak of carbon dioxide evolution, and reached up to $40 \mathrm{ppmv}$ at the end of a cycle. The integrated water vapor evolved during the desorption cycle was as low as $1 \mathrm{mg}$. Periods of high water concentration that were detected were shown to be experimental artifacts that could be eliminated in order to provide an accurate measurement. The pre-test laboratory work and alternation between sample and dry reference gas flows give confidence that the water vapor concentrations measured accurately represent conditions in the CDRA. All objectives of this test of the CDRA and the Mechanical Compressor Simulator system were achieved. 


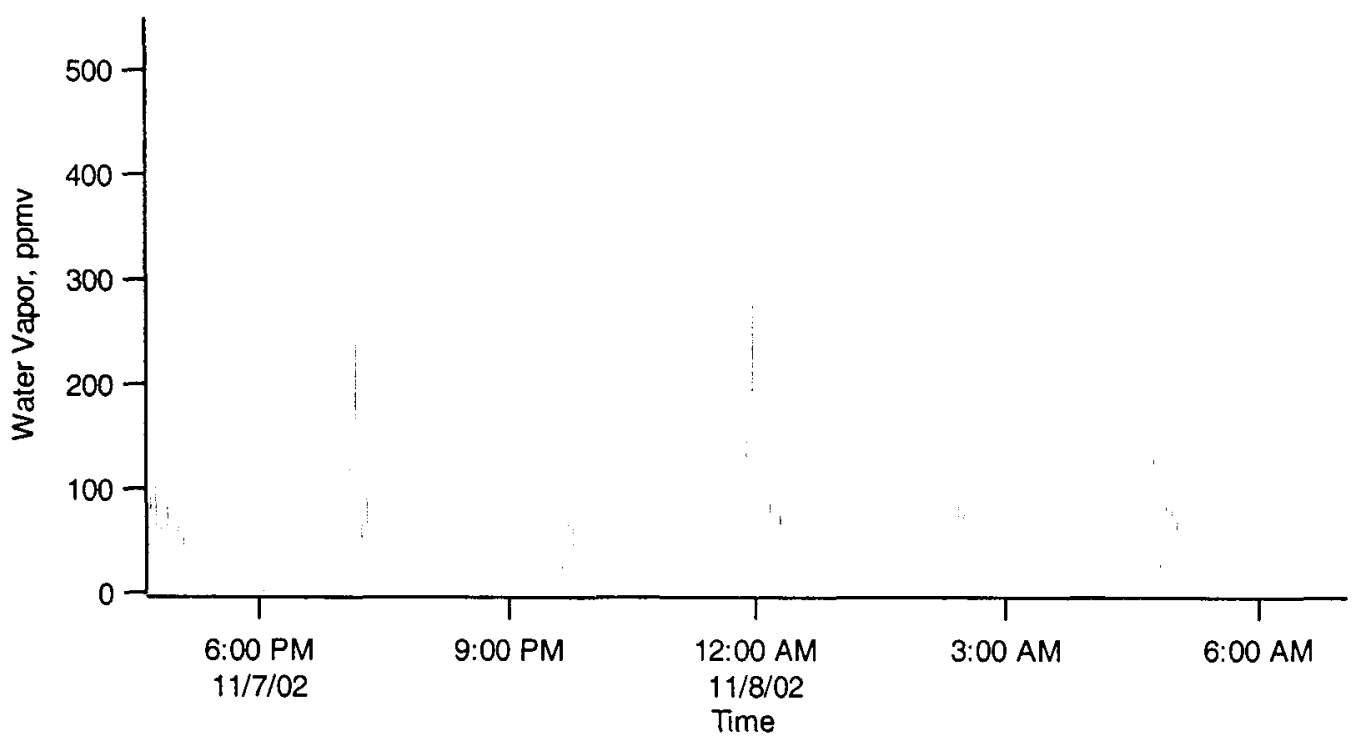

Figure 12. Water vapor record for overnight run with pump isolated at night.

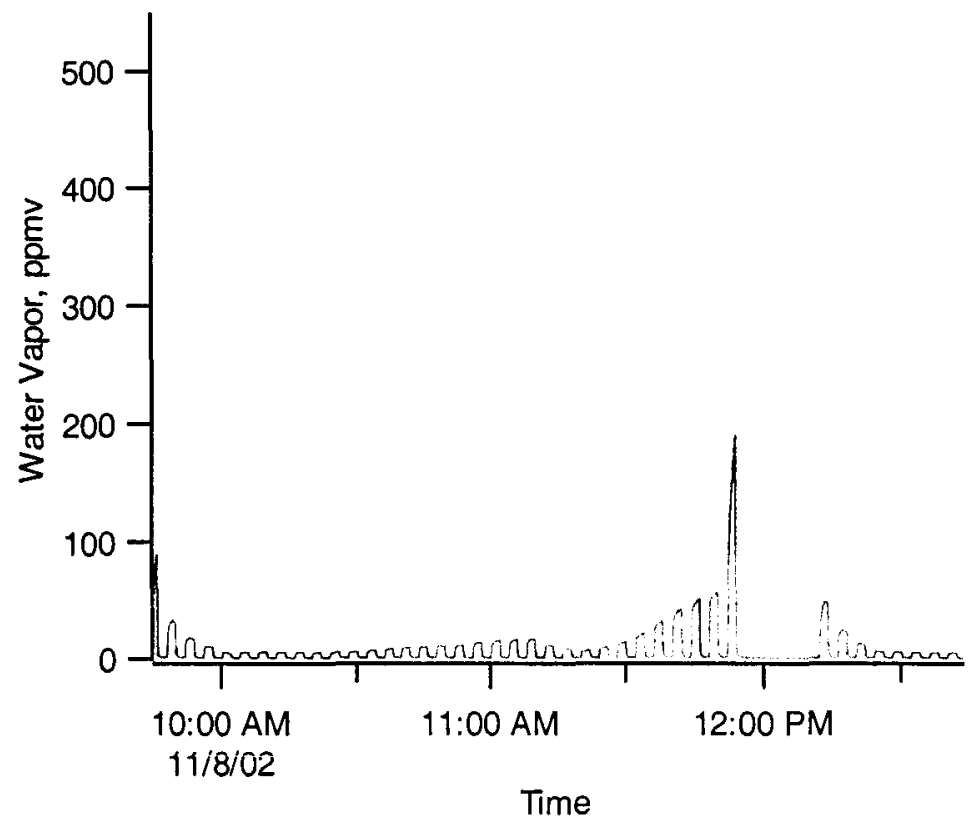

Figure 13. Water vapor record during operation without solar cycle, but with pump bypass valve closed

\section{REFERENCES}

1. Wormhoudt, J.C. and R.C. Miake-Lye, "Technical Support of Measurements of $\mathrm{SO}_{2}, \mathrm{SO}_{3}, \mathrm{HONO}, \mathrm{CO}_{2}$," Aerodyne Research Report RR-1362, prepared for NASA Glenn Research Center, 2003.

2. McManus, J.B., P.L. Kebabian and M.S. Zahniser, "Astigmatic Mirror Multiple Pass Absorption Cells for Long Pathlength Spectroscopy," Appl. Opt. 34, 33363348, 1995.

\section{CONTACT}

Joda Wormhoudt, Aerodyne Research, Inc., 45 Manning Road, Billerica, MA, 01821-3976, Tel: (978) 663-9500, Fax: (978) 663-4918. e-mail: jody@aerodyne.com. 